\title{
Measure of Noncompactness for Compact Matrix Operators on Some BK Spaces
}

\author{
E. Malkowsky ${ }^{1,2}$ and A. Alotaibi ${ }^{3}$ \\ ${ }^{1}$ Department of Mathematics, University of Giessen, Arndtstraße 2, 35392 Giessen, Germany \\ ${ }^{2}$ Department of Mathematics, Faculty of Arts and Sciences, Fatih University, Büyükçekmece, 34500 Istanbul, Turkey \\ ${ }^{3}$ Department of Mathematics, King Abdulaziz University, Jeddah 21589, Saudi Arabia
}

Correspondence should be addressed to A. Alotaibi; mathkerl1@hotmail.com

Received 20 May 2013; Accepted 31 October 2013; Published 10 February 2014

Academic Editor: Adrian Petrusel

Copyright (C) 2014 E. Malkowsky and A. Alotaibi. This is an open access article distributed under the Creative Commons Attribution License, which permits unrestricted use, distribution, and reproduction in any medium, provided the original work is properly cited.

\begin{abstract}
We study the spaces $w_{0}^{p}, w^{p}$, and $w_{\infty}^{p}$ of sequences that are strongly summable to 0 , summable, and bounded with index $p \geq 1$ by the Cesàro method of order 1 and establish the representations of the general bounded linear operators from the spaces $w^{p}$ into the spaces $w_{\infty}^{1}, w^{1}$, and $w_{0}^{1}$. We also give estimates for the operator norm and the Hausdorff measure of noncompactness of such operators. Finally we apply our results to characterize the classes of compact bounded linear operators from $w_{0}^{p}$ and $w^{p}$ into $w_{0}^{1}$ and $w^{1}$
\end{abstract}

\section{Introduction}

The spaces $w_{0}^{p}, w^{p}$, and $w_{\infty}^{p}$ of all complex sequences that are strongly summable to zero, strongly summable, and strongly bounded, with index $p \geq 1$ by the Cesàro method of order 1 , were first introduced and studied by Maddox $[1,2]$. Further recent studies on the spaces $w_{0}(p)$ where the constant index $p$ in $w_{0}^{p}$ is replaced by the terms of a positive sequence $p=\left(p_{k}\right)$ can be found in $[3,4]$. Extensive studies of generalizations of the spaces $w_{0}^{p}, w^{p}$, and $w_{\infty}^{p}$ to spaces of sequences of strong weighted means can be found in $[5,6]$.

In [7], a complete list was given of the characterizations of all matrix transformations from Maddox's spaces into the classical spaces $\ell_{\infty}, c, c_{0}$, and $\ell_{1}$ of all bounded, convergent, and null sequences and of all absolutely convergent series. The characterizations of matrix transformations from the classical sequence spaces into Maddox's spaces with index $p=1$ were established in [8]. Furthermore, some classes of compact bounded linear operators between those spaces were characterized.

Recently, several authors applied the Hausdorff measure of noncompactness to characterize matrix transformations between sequence spaces that are matrix domains of triangles in the classical sequence spaces, for instance, in [9-14].
In this paper, we extend our studies from the normally considered matrix transformations to the general bounded linear operators from $w^{p}$ into $w_{\infty}^{1}, w^{1}$, and $w_{0}^{1}$. We establish the representations of those operators, deduce estimates for their operator norms and Hausdorff measures of noncompactness, and characterize the corresponding classes of compact bounded linear operators.

\section{Notations and Basic Results}

In this section we list the notations, concepts, and basic results needed in the paper.

As usual, we denote by $\omega$ and $\phi$ the sets of all complex sequences $x=\left(x_{k}\right)_{k=1}^{\infty}$ and of all sequences that terminate in zeros; also let $e$ and $e^{(n)}$ for all $n \in \mathbb{N}$ be the sequences with $e_{k}=1$ for all $k$ and $e_{n}^{(n)}=0$ and $e_{k}^{(n)}=0$ for $k \neq n$.

A Banach space $X \subset \omega$ is a BK space if each coordinate $P_{n}: X \rightarrow \mathbb{C}$ with $P_{n} x=x_{n}$ for $x=\left(x_{k}\right)_{k=1}^{\infty} \in X$ is continuous. A BK space $X \supset \phi$ is said to have AK if $x^{[m]}=\sum_{k=1}^{m} x_{k} e^{(k)} \rightarrow x(m \rightarrow \infty)$ for every sequence $x=$ $\left(x_{k}\right)_{k=1}^{\infty} \in X$.

Let $(X,\|\cdot\|)$ be a normed space and $S_{X}=\{x \in X:\|x\|=1\}$ and $\bar{B}_{X}=\{x \in X:\|x\| \leq 1\}$ denote the unit sphere and closed 
unit ball in $X$, respectively. If $X$ and $Y$ are Banach spaces, then we write $\mathscr{B}(X, Y)$ for the space of all bounded linear operators $L: X \rightarrow Y$ with the operator norm $\|L\|=\sup \{\|L(x)\|: x \in$ $\left.S_{X}\right\}$; we write $X^{*}=\mathscr{B}(X, \mathbb{C})$ for the continuous dual of $X$, that is, the space of all continuous linear functionals on $X$ with the norm $\|f\|=\sup \left\{|f(x)|: x \in S_{X}\right\}$. Furthermore, if $(X,\|\cdot\|)$ is a normed sequence space, then we write $\|a\|_{X}^{*}=$ $\sup _{x \in S_{X}}\left|\sum_{k=1}^{\infty} a_{k} x_{k}\right|$ for $a \in \omega$ provided the expression on the right-hand side exists and is finite which is the case whenever $X$ is a BK space and $a \in X^{\beta}[15$, Theorem 7.2.9].

For any subset $X$ of $\omega$, the set $X^{\beta}=\left\{a \in \omega: \sum_{k=1}^{\infty} a_{k} x_{k}\right.$ converges for all $x \in X\}$ is called the $\beta$-dual of $X$.

Let $A=\left(a_{n k}\right)_{n, k=1}^{\infty}$ be an infinite matrix of complex numbers, let $X$ and $Y$ be subsets of $\omega$, and let $x \in \omega$. We write $A_{n}=\left(a_{n k}\right)_{k=1}^{\infty}$ for the sequence in the $n$th row of $A, A_{n} x=$ $\sum_{k=1}^{\infty} a_{n k} x_{k}, A x=\left(A_{n} x\right)_{n=1}^{\infty}$ (provided all the series converge) and $(X, Y)$ for the class of all matrices $A$ such that $A_{n} \in X^{\beta}$ for all $n \in \mathbb{N}$ and $A x \in Y$ for all $x \in X$. It is known that if $X$ and $Y$ are BK spaces then every matrix $A \in(X, Y)$ defines an operator $L_{A} \in \mathscr{B}(X, Y)$ by $L_{A}(x)=A x$ for all $x \in X$ [15, Theorem 4.2.8] and if, in addition, $X$ has AK then every operator $L \in \mathscr{B}(X, Y)$ is given by a matrix $A \in(X, Y)$ such that $L(x)=A x$ for all $x \in X[16$, Theorem 1.9].

Throughout, let $1 \leq p<\infty$, and let $q$ be the conjugate number of $p$; that is, $q=\infty$ for $p=1$ and $q=p /(p-1)$ for $1<p<\infty$. We write

$$
\begin{gathered}
w_{0}^{p}=\left\{x \in \omega: \lim _{n \rightarrow \infty} \frac{1}{n} \sum_{k=1}^{n}\left|x_{k}\right|^{p}=0\right\}, \\
w^{p}=\left\{x \in \omega: x-\xi \cdot e \in w_{0}^{p} \text { for some } \xi \in \mathbb{C}\right\}, \\
w_{\infty}^{p}=\left\{x \in \omega: \sup _{n} \frac{1}{n} \sum_{k=1}^{n}\left|x_{k}\right|^{p}<\infty\right\}
\end{gathered}
$$

for the sets of all sequences that are strongly summable to 0 , strongly summable, and strongly bounded, with index $p$ by the Cesàro method of order 1 ; if $p=1$, we write $w_{0}=w_{0}^{1}$, $w=w^{1}$, and $w_{\infty}=w_{\infty}^{1}$, for short.

The following results are known and can be found in [1] and, for instance, in [7, Proposition 1.1].

For each sequence $x \in w^{p}$, the $w^{p}$-limit $\xi$ for which

$$
x-\xi \cdot e \in w_{0}^{p}
$$

is unique. We write $\sum_{\nu}=\sum_{k=2^{v}}^{2^{v+1}-1}$ for $v=0,1, \ldots$ and $\max _{v}=$ $\max _{2^{v} \leq k \leq 2^{v+1}-1}$.

The sets $w_{0}^{p}, w^{p}$, and $w_{\infty}^{p}$ are BK space with the equivalent block and sectional norms

$$
\begin{aligned}
\|x\|_{b} & =\sup _{v \in \mathbb{N}_{0}}\left(\frac{1}{2^{v}} \sum_{\nu}\left|x_{k}\right|^{p}\right)^{1 / p}, \\
\|x\|_{s} & =\sup _{n}\left(\frac{1}{n} \sum_{k=1}^{n}\left|x_{k}\right|^{p}\right)^{1 / p} .
\end{aligned}
$$

$w_{0}^{p}$ is a closed subspace of $w^{p}$ and $w^{p}$ is a closed subspace of $w_{\infty}^{p} ; w_{0}^{p}$ has AK, and every sequence $x=\left(x_{k}\right)_{k=1}^{\infty} \in w^{p}$ has a unique representation

$$
x=\xi \cdot e+\sum_{k=1}^{\infty}\left(x_{k}-\xi\right) e^{(k)}
$$

where $\xi$ is the $w^{p}$-limit of $x$,

and $w_{\infty}^{p}$ has no Schauder basis. We always assume that $w_{0}^{p}$, $w^{p}$, and $w_{\infty}^{p}$ have the block norm, unless explicitly stated otherwise.

We put

$$
\|a\|_{\mathscr{M}_{p}}= \begin{cases}\sum_{\nu=0}^{\infty} 2^{v} \max _{\nu}\left|a_{k}\right| & (p=1) \\ \sum_{\nu=0}^{\infty} 2^{v / p}\left(\sum_{\nu}\left|a_{k}\right|^{q}\right)^{1 / q} & (1<p<\infty)\end{cases}
$$

and $\mathscr{M}_{p}=\left\{a \in \omega:\|a\|_{\mathscr{M}_{p}}<\infty\right\}$. The following results are known and can be found, for instance, in [7, Proposition 2.1]:

$$
\begin{gathered}
\left(w_{0}^{p}\right)^{\beta}=\left(w^{p}\right)^{\beta}=\left(w_{\infty}^{p}\right)^{\beta}=\mathscr{M}_{p}, \\
\left(w_{0}^{p}\right)^{*} \text { and } \mathscr{M}_{p} \text { are norm isomorphic, } \\
\|a\|_{w_{\infty}^{p}}^{*}=\|a\|_{\mathscr{M}_{p}} \quad \forall a \in w_{\infty}^{p} ;
\end{gathered}
$$

$f \in\left(w^{p}\right)^{*}$ if and only if there are $a_{0} \in \mathbb{C}$ and a sequence $a=\left(a_{k}\right)_{k=1}^{\infty} \in \mathscr{M}_{p}$ such that

$$
f(x)=\xi a_{0}+\sum_{k=1}^{\infty} a_{k} x_{k} \quad \forall x \in w^{p} \text { with } \xi \text { from (2). }
$$

Moreover

$$
\|f\|=\left|a_{0}\right|+\|a\|_{\mathscr{M}_{p}} \quad \forall f \in\left(w^{p}\right)^{*} .
$$

We need the following result where we assume that the initial and final spaces have the block and sectional norms, respectively.

Proposition 1. One has the following.

(a) $A \in\left(w_{\infty}^{p}, w_{\infty}\right)$ if and only if

$\|A\|_{\left(w_{\infty}^{p}, w_{\infty}\right)}=\sup _{m}\left(\frac{1}{m} \max _{N_{m} \subset\{1,2, \ldots, m\}}\left\|\sum_{n \in N_{m}} A_{n}\right\|_{\mathscr{M}_{p}}\right)<\infty$.

$$
\operatorname{Also}\left(w_{\infty}^{p}, w_{\infty}\right)=\left(w^{p}, w_{\infty}\right)=\left(w_{0}^{p}, w_{\infty}\right) .
$$

(b) $A \in\left(w_{0}^{p}, w_{0}\right)$ if and only if (11) holds and

$$
\lim _{m \rightarrow \infty} \frac{1}{m} \sum_{n=1}^{m}\left|a_{n k}\right|=0 \quad \text { for each } k \in \mathbb{N}
$$


(c) $A \in\left(w_{0}^{p}, w\right)$ if and only if (11) holds and

for each $k \in \mathbb{N}$ there exists $\alpha_{k} \in \mathbb{C}$ such that $\lim _{m \rightarrow \infty} \frac{1}{m} \sum_{n=1}^{m}\left|a_{n k}-\alpha_{k}\right|=0$.

(d) $A \in\left(w^{p}, w_{0}\right)$ if and only if (11) and (12) hold and

$$
\lim _{m \rightarrow \infty} \frac{1}{m} \sum_{n=1}^{m}\left|\sum_{k=1}^{\infty} a_{n k}\right|=0 .
$$

(e) $A \in\left(w^{p}, w\right)$ if and only if (11) and (13) hold and

$$
\lim _{m \rightarrow \infty} \frac{1}{m} \sum_{n=1}^{m}\left|\sum_{k=1}^{\infty} a_{n k}-\tilde{\alpha}\right|=0 \quad \text { for some } \tilde{\alpha} \in \mathbb{C} \text {. }
$$

(f) If $A \in(X, Y)$ in the cases above, then

$$
\|A\|_{\left(w_{\infty}^{p}, w_{\infty}\right)} \leq\left\|L_{A}\right\| \leq 4 \cdot\|A\|_{\left(w_{\infty}^{p}, w_{\infty}\right)} .
$$

Proof. (a) follows from [17, Corollary 1], (6), and (7).

(b) and (c) follow from $[15,8.3 .6, \mathrm{p} .123]$, since $w_{0}^{p}$ and $w^{p}$ are closed subspaces of $w_{\infty}^{p}$.

(d) and (e) follow from Parts (b) and (c) by [15, 8.3.7].

(f) follows from [17, equation (2.8)], (6), and (7).

\section{Representation of Bounded Linear Operators}

Here we establish the representations of the bounded linear operators in $\mathscr{B}\left(w^{p}, Y\right)$ for $Y=w_{\infty}, w, w_{0}$ and give estimates for the operator norms in each case. Throughout, we assume that $w^{p}$ and $Y$ have the block and sectional norms, respectively.

We note that, since $w_{0}^{p}$ has $\mathrm{AK}$, every $L \in \mathscr{B}\left(w_{0}^{p}, Y\right)$ is given by an infinite matrix $A \in\left(w_{0}^{p}, Y\right)$, and its operator norm satisfies the inequalities in (16).

Theorem 2. (a) One has $L \in \mathscr{B}\left(w^{p}, w_{\infty}\right)$ if and only if there exist a matrix $A \in\left(w_{0}^{p}, w_{\infty}\right)$ and a sequence $b \in w_{\infty}$ such that

$$
L(x)=b \cdot \xi+A x \quad \forall x \in w^{p},
$$$$
\text { where } \xi \text { is the } w^{p} \text {-limit of } x \text {. }
$$

Moreover, one has

$$
\begin{aligned}
& \sup _{m}\left(\frac{1}{m} \max _{N_{m} \subset\{1, \ldots, m\}}\left(\left|\sum_{n \in N_{m}} b_{n}\right|+\left\|\sum_{n \in N_{m}} A_{n}\right\| \|_{\mathscr{M}_{p}}\right)\right) \\
& \leq\|L\| \leq 4 \cdot \sup _{m}\left(\frac{1}{m} \max _{N_{m} \subset\{1, \ldots, m\}}\left(\left|\sum_{n \in N_{m}} b_{n}\right|+\left\|\sum_{n \in N_{m}} A_{n}\right\|_{\mathscr{M}_{p}}\right)\right) .
\end{aligned}
$$

(b) One has $L \in \mathscr{B}\left(w^{p}, w\right)$ if and only if there exist a matrix $A \in\left(w_{0}^{p}, w\right)$ and a sequence $b \in w_{\infty}$ with

$$
\lim _{m \rightarrow \infty} \frac{1}{m} \sum_{n=1}^{m}\left|b_{n}+A_{n} e-\tilde{\beta}\right|=0 \quad \text { for some } \tilde{\beta} \in \mathbb{C}
$$

such that (17) holds; moreover, one has (18).

(c) One has $L \in \mathscr{B}\left(w^{p}, w_{0}\right)$ if and only if there exist a matrix $A \in\left(w_{0}^{p}, w_{0}\right)$ and a sequence $b \in w_{\infty}$ with

$$
\lim _{m \rightarrow \infty} \frac{1}{m} \sum_{n=1}^{m}\left|b_{n}+A_{n} e\right|=0
$$

such that (17) holds; moreover, one has (18).

Proof. (a) First we assume $L \in \mathscr{B}\left(w^{p}, w_{\infty}\right)$ and write $L_{n}=$ $P_{n} \circ L$ for $n=1,2, \ldots$ where $P_{n}$ denotes the $n$th coordinate. Since $w^{p}$ is a $B K$ space, it follows that $L_{n} \in\left(w^{p}\right)^{*}$ for each $n$, and hence we have by (9)

$$
L_{n}(x)=b_{n} \cdot \xi+A_{n} x \quad \forall x \in w^{p},
$$

where $\xi$ is the $w^{p}$-limit of $x$,

$$
\begin{gathered}
b_{n}=L_{n}(e)-\sum_{k=1}^{\infty} L_{n}\left(e^{(k)}\right), \\
a_{n k}=L_{n}\left(e^{(k)}\right) \quad \forall n \text { and } k, \\
A_{n} \in \mathscr{M}_{p} \quad \forall n .
\end{gathered}
$$

This yields (17); moreover, we have by (10)

$$
\left\|L_{n}\right\|=\left|b_{n}\right|+\left\|A_{n}\right\|_{\mathscr{M}_{p}} \quad \text { for } n=1,2, \ldots
$$

It also follows from $(21)$ and $L(e) \in w_{\infty}$ that

$$
\sup _{m} \frac{1}{m} \sum_{n=1}^{m}\left|L_{n}(e)\right|=\sup _{m} \frac{1}{m} \sum_{n=1}^{m}\left|b_{n}+A e\right|<\infty,
$$

and so $b+A e \in w_{\infty}$. Furthermore, since $L\left(x^{(0)}\right)=A x^{(0)}$ for all $x^{(0)} \in w_{0}^{p}$, we have $A \in\left(w_{0}^{p}, w_{\infty}\right)=\left(w_{\infty}^{p}, w_{\infty}\right)$, and so $A e \in w_{\infty}$ and we obtain $b=(b+A e)-A e \in w_{\infty}$.

Now we show (18). We define $L_{N_{m}}: w^{p} \rightarrow \mathbb{C}$ for all $m \in \mathbb{N}$ and for each subset $N_{m}$ of $\{1,2, \ldots, m\}$ by

$$
L_{N_{m}}=\frac{1}{m} \sum_{m \in N_{m}} L_{n}
$$

Then clearly $L_{N_{m}} \in\left(w^{p}\right)^{*}$, and we obtain by a well-known inequality (cf. [18])

$$
\left|L_{N_{m}}(x)\right| \leq \frac{1}{m} \sum_{n=1}^{m}\left|L_{n}(x)\right| \leq 4 \cdot \max _{N_{m} \subset\{1, \ldots, m\}}\left|L_{N_{m}}(x)\right|,
$$

and hence by the first inequality in (26) and by (10)

$$
\begin{aligned}
\left\|L_{N_{m}}\right\| & =\frac{1}{m}\left\|\sum_{n \in N_{m}} L_{n}\right\| \\
& =\frac{1}{m}\left(\left|\sum_{n \in N_{m}} b_{n}\right|+\left\|\sum_{n \in N_{m}} A_{n}\right\|_{\mathscr{M}_{p}}\right) \leq\|L\|
\end{aligned}
$$


for all $m \in \mathbb{N}$ and all $N_{m} \subset\{1,2, \ldots, m\}$, and so the first inequality in (18) follows. Also, we obtain for all $m \in \mathbb{N}$ from the second inequality in (26) and by (10)

$$
\begin{aligned}
& \frac{1}{m} \sum_{n=1}^{m}\left|L_{n}(x)\right| \\
& \leq 4 \cdot \max _{N_{m} \subset\{1, \ldots, m\}}\left\|L_{N_{m}}\right\| \\
& \leq 4 \cdot \sup _{m}\left(\frac{1}{m} \max _{N_{m} \subset\{1, \ldots, m\}}\left(\left|\sum_{n \in N_{m}} b_{n}\right|+\left\|\sum_{n \in N_{m}} A_{n}\right\|_{\mathscr{M}_{p}}\right)\right) .
\end{aligned}
$$

This implies the second inequality in (18).

Conversely, we assume that $A \in\left(w_{0}^{p}, w_{\infty}\right)$, that $b \in w_{\infty}$, and that (17) is satisfied. Let $\varepsilon>0$, and $x \in w^{p}$ be given and let $\xi$ be the $w^{p}$-limit of $\xi$. Then there exists $M \in \mathbb{N}$ such that $(1 / m) \sum_{n=1}^{\infty}\left|x_{k}-\xi\right|^{p}<\varepsilon^{p}$ for all $m>M$. Thus we have for all $m>M$

$$
\begin{aligned}
|\xi| & =\left(\frac{1}{m} \sum_{n=1}^{m}|\xi|^{p}\right)^{1 / p} \\
& \leq\left(\frac{1}{m} \sum_{n=1}^{m}\left|x_{n}\right|^{p}\right)^{1 / p}+\left(\frac{1}{m} \sum_{n=1}^{m}\left|x_{n}-\xi\right|^{p}\right)^{1 / p} \\
& \leq\|x\|_{b}+\varepsilon .
\end{aligned}
$$

Since $\varepsilon$ was arbitrary, we have

$$
|\xi| \leq\|x\|_{b}
$$

We define the map $g: w^{p} \rightarrow w_{\infty}$ with $g(x)=b \xi$ for all $x \in$ $w^{p}$, where $\xi \in \mathbb{C}$ is the $w^{p}$-limit of $x$. Then $g$ trivially is linear, and it follows from (30) that

$$
\|g(x)\|_{b}=\sup _{m} \frac{1}{m} \sum_{n=1}^{m}\left|b_{n} \xi\right| \leq \sup _{m}\left(\frac{1}{m} \sum_{n=1}^{m}\left|b_{n}\right|\right) \cdot\|x\|_{b},
$$

and, since $b \in w_{\infty}$, we obtain $g \in \mathscr{B}\left(w^{p}, w_{\infty}\right)$. Furthermore, we have $A \in\left(w_{0}^{p}, w_{\infty}\right)=\left(w^{p}, w_{\infty}\right)$, and hence $L_{A} \in$ $\mathscr{B}\left(w^{p}, w_{\infty}\right)$, and so, by (17), $L=g+L_{A} \in \mathscr{B}\left(w^{p}, w_{\infty}\right)$.

(b) First we assume $L \in \mathscr{B}\left(w^{p}, w\right)$. Then $L \in \mathscr{B}\left(w^{p}, w_{\infty}\right)$, and by Part (a) there are $b \in w_{\infty}$ and $A \in\left(w_{0}^{p}, w_{\infty}\right)=$ $\left(w^{p}, w_{\infty}\right)$ such that (17) is satisfied; also clearly (18) is satisfied. It follows from (17), $L(e) \in w$, and $L\left(e^{(k)}\right) \in w$ for each $k$ that there exist $\widetilde{\beta} \in \mathbb{C}$ and $\alpha_{k} \in \mathbb{C}$ for $k=1,2, \ldots$ such that

$$
\lim _{m \rightarrow \infty} \frac{1}{m} \sum_{n=1}^{m}\left|L_{n}(e)-\tilde{\beta}\right|=\lim _{m \rightarrow \infty} \frac{1}{m} \sum_{n=1}^{m}\left|b_{n}+A_{n} e-\tilde{\beta}\right|=0,
$$

which is (19), and

$$
\begin{aligned}
\lim _{m \rightarrow \infty} & \frac{1}{m} \sum_{n=1}^{m}\left|L_{n}\left(e^{(k)}\right)-\alpha_{k}\right| \\
= & \lim _{m \rightarrow \infty} \frac{1}{m} \sum_{n=1}^{m}\left|a_{n k}-\alpha_{k}\right|=0 \text { for each } k .
\end{aligned}
$$

Now it follows from $A \in\left(w_{0}^{p}, w_{\infty}\right)$ and (33) by Proposition 1(c) that $A \in\left(w_{0}^{p}, w\right)$.

Conversely, we assume that $A \in\left(w_{0}^{p}, w\right)$, that $b \in w_{\infty}$, and that (17) and (19) are satisfied. Then we have $A \in\left(w_{0}^{p}, w_{\infty}\right)$, and so $L \in \mathscr{B}\left(w^{p}, w_{\infty}\right)$ by Part (a). Let $x \in w^{p}$ be given and let $\xi$ be the $w^{p}$-limit of $x$. Then we have $x^{(0)}=x-\xi e \in w_{0}^{p}$ and, by (17),

$$
L_{n}(x)=b_{n} \xi+\sum_{k=1}^{\infty} a_{n k} x_{k}=\left(b_{n}+A_{n} e\right) \xi+A_{n} x^{(0)}
$$

Since $A \in\left(w_{0}^{p}, w\right)$, the $w$-limit of $\eta_{0}$ of $A x^{(0)}$ exists and we have by (19) and (34)

$$
\begin{aligned}
0 & \leq \lim _{m \rightarrow \infty}\left(\frac{1}{m} \sum_{n=1}^{m}\left|L_{n}(x)-\left(\widetilde{\beta} \xi+\eta_{0}\right)\right|\right) \\
\leq & \lim _{m \rightarrow \infty}\left(\frac{1}{m} \sum_{n=1}^{m}\left|\left(b_{n}+A_{n} e-\widetilde{\beta}\right) \cdot \xi\right|\right) \\
& +\lim _{m \rightarrow \infty}\left(\frac{1}{m} \sum_{n=1}^{m}\left|A_{n} x^{(0)}-\eta_{0}\right|\right) \\
= & |\xi| \cdot \lim _{m \rightarrow \infty}\left(\frac{1}{m} \sum_{n=1}^{m}\left|b_{n}+A_{n} e-\tilde{\beta}\right|\right)=0 .
\end{aligned}
$$

Therefore we have $L \in \mathscr{B}\left(w^{p}, w\right)$.

(c) The proof of Part (c) is similar to that of Part (b) with $\tilde{\beta}=0$ and $\alpha_{k}=0(k=1,2, \ldots)$.

Remark 3. It was shown in the proof of [19, Theorem 3.6] that if $A \in\left(w_{0}^{p}, w\right)$ then $\left(\alpha_{k}\right)_{k=1}^{\infty} \in \mathscr{M}_{p}$ with $\alpha_{k}(k=1,2, \ldots)$ from (33) and in [19, equation (3.14)] that the $w$-limit of $A x^{(0)}$ for any sequence $x^{(0)}$ in $w_{0}^{p}$ is given by

$$
\eta_{0}=\sum_{k=1}^{\infty} \alpha_{k} x_{k}^{(0)}
$$

Let $L \in \mathscr{B}\left(w^{p}, w\right)$ and $x \in w^{p}$ and let $\xi$ be the $w^{p}$-limit of $x$; then we obtain by (35) and (36) for the $w$-limit of $L(x)$

$$
\begin{aligned}
\eta & =\tilde{\beta} \cdot \xi+\eta_{0}=\tilde{\beta} \cdot \xi+\sum_{k=1}^{\infty} \alpha_{k}\left(x_{k}-\xi\right) \\
& =\left(\widetilde{\beta}-\sum_{k=1}^{\infty} \alpha_{k}\right) \cdot \xi+\sum_{k=1}^{\infty} \alpha_{k} x_{k} \quad \text { with } \widetilde{\beta} \text { from (19). }
\end{aligned}
$$

\section{Compact Operators}

In this section, we establish estimates for the Hausdorff measures of noncompactness of linear operators and characterize some classes of compact operators from $X$ into $Y$, where $X=w_{0}^{p}, w^{p}$ and $Y=w_{0}, w$.

First we recall some useful definitions and results. The reader is referred to the monographs [20-23] for the theory and applications of measures of noncompactness. Let $X$ and 
$Y$ be Banach spaces and let $L: X \rightarrow Y$ be a linear operator. Then $L$ is said to be compact if its domain is all of $X$ and, for every bounded sequence $\left(x_{n}\right)_{n=1}^{\infty}$ in $X$, the sequence $\left(L\left(x_{n}\right)\right)_{n=1}^{\infty}$ has a convergent subsequence in $Y$. We denote the class of such operators by $\mathscr{C}(X, Y)$.

Let $(X, d)$ be a metric space, $B(x, r)=\{y \in X: d(y, x)<$ $r$ \} denote the open ball of radius $r$ and centre in $x$, and $\mathscr{M}_{X}$ denote the class of bounded subsets of $M$. Then the map $\chi$ : $\mathscr{M}_{X} \rightarrow[0, \infty)$ with

$$
\begin{aligned}
\chi(Q)=\inf \left\{\varepsilon>0: Q \subset \bigcup_{k=1}^{n} B\left(x_{k}, r_{k}\right),\right. \\
\left.\quad x_{k} \in X, r_{k}<\varepsilon(k=1, \ldots, n ; n \in \mathbb{N})\right\}
\end{aligned}
$$

is called the Hausdorff measure of noncompactness.

Let $X$ and $Y$ be Banach spaces and let $\chi_{1}$ and $\chi_{2}$ be the Hausdorff measures of noncompactness on $X$ and $Y$. Then the operator $L: X \rightarrow Y$ is said to be $\left(\chi_{1}, \chi_{2}\right)$-bounded if $L(Q) \in \mathscr{M}_{Y}$ for every $Q \in \mathscr{M}_{X}$, and there exists a positive constant $C$ such that $\chi_{2}(L(Q)) \leq C \cdot \chi_{1}(Q)$ for every $Q \in \mathscr{M}_{X}$. If an operator $L$ is $\left(\chi_{1}, \chi_{2}\right)$-bounded, then

$$
\begin{gathered}
\|L\|_{\left(\chi_{1}, \chi_{2}\right)}=\inf \left\{C>0: \chi_{2}(L(Q)) \leq C \cdot \chi_{1}(Q)\right. \\
\left.\forall Q \in \mathscr{M}_{X}\right\}
\end{gathered}
$$

is called the $\left(\chi_{1}, \chi_{2}\right)$-measure of noncompactness of $L$. In particular, if $\chi_{1}=\chi_{2}=\chi$, then we write $\|L\|_{\chi}=\|L\|_{(\chi, \chi)}$.

The following useful results are well known.

Proposition 4. Let $X$ and $Y$ be Banach spaces and $L \in$ $\mathscr{B}(X, Y)$. Then one has

$$
\|L\|_{\chi}=\chi\left(L\left(\bar{B}_{X}\right)\right)=\chi\left(L\left(S_{X}\right)\right)
$$

(see [23, Theorem 2.25]),

$$
L \in \mathscr{C}(X, Y) \text { iff }\|L\|_{\chi}=0
$$

(see [23, Corollary 2.26, equation (2.58)]).

We also need the following results which are an immediate consequence of [8, Proposition 3.2 and Lemma 3.5].

Proposition 5. (a) Let $\mathscr{P}_{n}: w \rightarrow w$ be the projectors onto the linear span of $\left\{e, e^{(1)}, \ldots, e^{(n)}\right\}, I: w \rightarrow w$ the identity, and $\mathscr{R}_{n}=I-\mathscr{P}_{n}$ for $n=0,1, \ldots$. Then one has for all $Q \in \mathscr{M}_{w}$

$$
\frac{1}{2} \cdot \lim _{n \rightarrow \infty}\left(\sup _{x \in Q}\left\|\mathscr{R}_{n}(x)\right\|\right) \leq \chi(Q) \leq \lim _{n \rightarrow \infty}\left(\sup _{x \in Q}\left\|\mathscr{R}_{n}(x)\right\|\right) .
$$

(b) Let $\mathscr{P}_{n}: w_{0} \rightarrow w_{0}$ be the projectors onto the linear span of $\left\{e^{(1)}, e^{(2)}, \ldots, e^{(n)}\right\}, I: w_{0} \rightarrow w_{0}$ the identity, and $\mathscr{R}_{n}=I-\mathscr{P}_{n}$ for $n=0,1, \ldots$. Then one has for all $Q \in \mathscr{M}_{w_{0}}$

$$
\chi(Q)=\lim _{n \rightarrow \infty}\left(\sup _{x \in Q}\left\|\mathscr{R}_{n}(x)\right\|\right) .
$$

Proof. (a) The inequalities in (42) follow from [8, Proposition 3.2 and Lemma 3.5].

(b) The identity in (42) follows from [8, Proposition 3.2 and Lemma 3.3(a)].

Now we give estimates for the Hausdorff measures of noncompactness of the general operators $L \in \mathscr{B}\left(w^{p}, w\right)$ and $L \in \mathscr{B}\left(w^{p}, w_{0}\right)$. Let $m, r \in \mathbb{N}$ and $m>r$. Then we write $N(m, r)$ for any subset of the set $\{r+1, r+2, \ldots, m\}$.

Theorem 6. (a) Let $L \in \mathscr{B}\left(w^{p}, w\right)$. One uses the notations of Theorem 2 and writes $\gamma_{n}=b_{n}-\widetilde{\beta}+\sum_{k=1}^{\infty} \alpha_{k}$ for $n=1,2, \ldots$ and $C=\left(c_{n k}\right)_{n, k=1}^{\infty}$ for the matrix with $c_{n k}=a_{n k}-\alpha_{k}$ for all $n$ and $k$. Then one has

$$
\begin{array}{r}
\frac{1}{2} \lim _{r \rightarrow \infty}\left(\sup _{m}\left(\frac{1}{m} \max _{N(m, r)}\left(\left|\sum_{n \in N(m, r)} \gamma_{n}\right|+\mid \sum_{n \in N(m, r)} C_{n} \|_{\mathscr{M}_{p}}\right)\right)\right) \\
\leq\|L\|_{\chi} \leq 4 \cdot \lim _{r \rightarrow \infty}\left(\operatorname { s u p } _ { m } \left(\frac { 1 } { m } \operatorname { m a x } _ { N ( m , r ) } \left(\left|\sum_{n \in N(m, r)} \gamma_{n}\right|\right.\right.\right. \\
\left.\left.\left.+\| \sum_{n \in N(m, r)} C_{n}\right)\right)\right) .
\end{array}
$$

(b) Let $L \in \mathscr{B}\left(w^{p}, w_{0}\right)$. Then one has

$$
\begin{array}{r}
\lim _{r \rightarrow \infty}\left(\sup _{m}\left(\frac{1}{m} \max _{N(m, r)}\left(\left|\sum_{n \in N(m, r)} b_{n}\right|+|| \sum_{n \in N(m, r)} A_{n} \|_{\mathscr{M}_{p}}\right)\right)\right) \\
\leq\|L\|_{\chi} \leq 4 \cdot \lim _{r \rightarrow \infty}\left(\operatorname { s u p } _ { m } \left(\frac { 1 } { m } \operatorname { m a x } _ { N ( m , r ) } \left(\left|\sum_{n \in N(m, r)} b_{n}\right|\right.\right.\right. \\
\left.\left.\left.+\left\|\sum_{n \in N(m, r)} A_{n}\right\|_{\mathscr{M}_{p}}\right)\right)\right) .
\end{array}
$$

Proof. (a) We assume $L \in \mathscr{B}\left(w^{p}, w\right)$.

Let $x \in w^{p}$ be given, $\xi$ be the $w^{p}$-limit of $x$, and $y=L(x)$. Then we have from Theorem 2(b) and (17) that $y=b \cdot \xi+A x$, where $A \in\left(w_{0}^{p}, w\right)$ and $b \in w_{\infty}$, and it follows that

$$
\begin{aligned}
y_{n} & =b_{n} \cdot \xi+A_{n} x \\
& =\left(b_{n}+\sum_{k=1}^{\infty} a_{n k}\right) \cdot \xi+A_{n}(x-\xi \cdot e) \quad \forall n \in \mathbb{N} .
\end{aligned}
$$

Furthermore, the complex numbers $\widetilde{\beta}$ and $\alpha_{k}(k=1,2, \ldots)$ satisfying (19) and (33) exist by Theorem 2(b), and $\left(\alpha_{k}\right)_{k=1}^{\infty} \epsilon$ $\mathscr{M}_{p}$ by Remark 3, so $\eta \in \mathbb{C}$ defined in (37) is the $w$-limit of $y=L(x)$ by Remark 3. Now let $\mathscr{P}_{r}: w \rightarrow w$ be the projector onto the linear span of $\left\{e, e^{(1)}, \ldots, e^{(r)}\right\}$ and $\mathscr{R}_{r}=$ $I-\mathscr{P}_{r}$ for $r=0,1, \ldots$, where $I$ is the identity on $w$; hence 
$\mathscr{R}_{r}(y)=\sum_{n=r+1}^{\infty}\left(y_{n}-\eta\right) e^{(n)}$ by (4). Let $r \in \mathbb{N}$ be given. We write $f_{n, r}(x)=\left(\mathscr{R}_{r}(L(x))\right)_{n}$ for all $n$ and obtain $f_{n, r}(x)=0$ for $n \leq r$ and, for $n>r$, from (46) and (37)

$f_{n, r}(x)$

$=y_{n}-\eta=b_{n} \cdot \xi+A_{n} x-\left(\tilde{\beta} \cdot \xi+\sum_{k=1}^{\infty} \alpha_{k}\left(x_{k}-\xi\right)\right)$

$=\left(b_{n}-\tilde{\beta}+\sum_{k=1}^{\infty} \alpha_{k}\right) \cdot \xi+\sum_{k=1}^{\infty}\left(a_{n k}-\alpha_{k}\right) x_{k}=\gamma_{n} \cdot \xi+C_{n} x$.

Since $f_{n, r} \in\left(w^{p}\right)^{*}$, we obtain by the same kind of argument as in the proof of (18) that

$$
\begin{aligned}
& \sup _{m}\left(\frac{1}{m} \max _{N(m, r)}\left(\left|\sum_{n \in N(m, r)} \gamma_{n}\right|+\left\|\sum_{n \in N(m, r)} C_{n}\right\|_{\mathscr{M}_{p}}\right)\right) \\
& \leq \sup _{x \in S_{w^{p}}}\left\|\mathscr{R}_{r}(L(x))\right\| \\
& \leq 4 \cdot \sup _{m}\left(\frac{1}{m} \max _{N(m, r)}\left(\left|\sum_{n \in N(m, r)} \gamma_{n}\right|+\left\|\sum_{n \in N(m, r)} C_{n}\right\|_{\mathscr{M}_{p}}\right)\right) .
\end{aligned}
$$

Now the inequalities in (44) follow from (40) and (42).

(b) Now $\widetilde{\beta}=\alpha_{k}=0$ for $k=1,2, \ldots$, and as in the proof of Part (a) we obtain (48) with $\gamma_{n}$ and $C_{n}$ replaced by $b_{n}$ and $A_{n}$, respectively, and the inequalities in (45) follow from (40) and (43).

Corollary 7. (a) Let $L \in \mathscr{B}\left(w_{0}^{p}, w\right)$. Then $L$ is given by a matrix $A \in\left(w_{0}^{p}, w\right)$ and one has, writing $C=\left(c_{n k}\right)_{n, k=1}^{\infty}$ for the matrix with $c_{n k}=a_{n k}-\alpha_{k}$ for all $n$ and $k$, where $\alpha_{k}$ is given by (33),

$$
\begin{aligned}
& \frac{1}{2} \lim _{r \rightarrow \infty}\left(\sup _{m}\left(\frac{1}{m} \max _{N(m, r)}\left\|\sum_{n \in N(m, r)} C_{n}\right\|_{\mathscr{M}_{p}}\right)\right) \\
& \leq\|L\|_{\chi} \leq 4 \cdot \lim _{r \rightarrow \infty}\left(\sup _{m}\left(\frac{1}{m} \max _{N(m, r)}\left\|\sum_{n \in N(m, r)} C_{n}\right\|_{\mathscr{M}_{p}}\right)\right) .
\end{aligned}
$$

(b) Let $L \in \mathscr{B}\left(w_{0}^{p}, w_{0}\right)$. Then one has

$$
\begin{aligned}
& \lim _{r \rightarrow \infty}\left(\sup _{m}\left(\frac{1}{m} \max _{N(m, r)}\left\|\sum_{n \in N(m, r)} A_{n}\right\|_{\mathscr{M}_{p}}\right)\right) \\
& \leq\|L\|_{\chi} \leq 4 \cdot \lim _{r \rightarrow \infty}\left(\sup _{m}\left(\frac{1}{m} \max _{N(m, r)}\left\|\sum_{n \in N(m, r)} A_{n}\right\|_{\mathscr{M}_{p}}\right)\right) .
\end{aligned}
$$

TABLE 1

\begin{tabular}{lccc}
\hline To & & From & \\
& $w_{0}^{p}$ & & $w^{p}$ \\
\hline$w_{0}$ & $(1)$ & $(2)$ \\
$w$ & $(3)$ & $(4)$ \\
\hline
\end{tabular}

Proof. (a) The estimates in (49) are easily obtained from [8, Theorem 3.6] with $\|\cdot\|_{\left(X, w_{\infty}\right)}^{*}=\|\cdot\|_{\left(w_{\infty}^{p}, w_{\infty}\right)}$ defined in (11).

(b) The estimates in (50) follow from those in (45) with $b_{n}=0$ for all $n$.

We apply our results and close with the characterizations of the classes $\mathscr{C}(X, Y)$ for $X=w^{p}, w_{0}^{p}$ and $Y=w, w_{0}$.

Corollary 8. Let $L \in \mathscr{B}(X, Y)$. Then the necessary and sufficient conditions for $L \in \mathscr{C}(X, Y)$ can be read from Table 1 where

$$
\begin{aligned}
& \text { (1) } \lim _{r \rightarrow \infty}\left(\sup _{m}\left(\frac{1}{m} \max _{N(m, r)}\left\|\sum_{n \in N(m, r)} A_{n}\right\|_{\mathscr{M}_{p}}\right)\right)=0 \text {, } \\
& \text { (2) } \lim _{r \rightarrow \infty}\left(\operatorname { s u p } _ { m } \left(\frac { 1 } { m } \operatorname { m a x } _ { N ( m , r ) } \left(\left|\sum_{n \in N(m, r)} b_{n}\right|\right.\right.\right. \\
& \left.\left.+\left\|\sum_{n \in N(m, r)} A_{n}\right\|_{\mathscr{M}_{p}}\right)\right)=0, \\
& \text { (3) } \lim _{r \rightarrow \infty}\left(\sup _{m}\left(\frac{1}{m} \max _{N(m, r)}\left\|\sum_{n \in N(m, r)} C_{n}\right\|_{\mathscr{M}_{p}}\right)\right)=0 \text {, } \\
& \text { (4) } \lim _{r \rightarrow \infty}\left(\operatorname { s u p } _ { m } \left(\frac { 1 } { m } \operatorname { m a x } _ { N ( m , r ) } \left(\left|\sum_{n \in N(m, r)} \gamma_{n}\right|\right.\right.\right. \\
& \left.\left.+\left\|\sum_{n \in N(m, r)} C_{n}\right\|_{\mathscr{M}_{p}}\right)\right)=0
\end{aligned}
$$

Proof. The conditions in (1)-(4) are immediate consequences of (41) and the conditions in (50), (45), (49), and (44), respectively.

\section{Conflict of Interests}

The authors declare that there is no conflict of interests regarding the publication of this paper.

\section{Acknowledgment}

The authors gratefully acknowledge the financial support from King Abdulaziz University, Jeddah, Saudi Arabia. 


\section{References}

[1] I. J. Maddox, “On Kuttner's theorem," Journal of the London Mathematical Society, vol. 43, pp. 285-290, 1968.

[2] I. J. Maddox, Infinite Matrices of Operators, vol. 786 of Lecture Notes in Mathematics, Springer, Berlin, Germany, 1980.

[3] P. Spanos and B. Thorpe, "The $w_{0}(p)-w_{0}(q)$ mapping problem for factorable matrices. II," Journal of Mathematical Analysis and Applications, vol. 341, no. 1, pp. 689-693, 2008.

[4] P. Spanos and B. Thorpe, "The $w_{0}(p)-w_{0}(q)$ mapping problem for factorable matrices. I," Journal of Mathematical Analysis and Applications, vol. 341, no. 2, pp. 751-763, 2008.

[5] K.-G. Grosse-Erdmann, The Blocking Technique, Weighted Mean Operators and Hardy's Inequality, vol. 1679 of Lecture Notes in Mathematics, Springer, Berlin, Germany, 1998.

[6] K.-G. Grosse-Erdmann, "Strong weighted mean summability and Kuttner's theorem," Journal of the London Mathematical Society, vol. 59, no. 3, pp. 987-1002, 1999.

[7] F. Başar, E. Malkowsky, and B. Altay, "Matrix transformations on the matrix domains of triangles in the spaces of strongly $C_{1}$ summable and bounded sequences," Publicationes Mathematicae Debrecen, vol. 73, no. 1-2, pp. 193-213, 2008.

[8] I. Djolović and E. Malkowsky, "Compact operators into the spaces of strongly $C_{1}$ summable and bounded sequences," Nonlinear Analysis. Theory, Methods \& Applications, vol. 74, no. 11, pp. 3736-3750, 2011.

[9] M. Başarır and E. E. Kara, "On the B-difference sequence space derived by generalized weighted mean and compact operators," Journal of Mathematical Analysis and Applications, vol. 391, no. 1, pp. 67-81, 2012.

[10] M. Basarir and E. E. Kara, "On compact operators on the Riesz $B^{m}$-difference sequence space," Iranian Journal of Science and Technology. Transaction A, vol. 35, no. 4, pp. 279-285, 2011.

[11] I. Djolović, "Compact operators on the spaces $a_{0}^{r}(\Delta)$ and $a_{c}^{r}(\Delta)$," Journal of Mathematical Analysis and Applications, vol. 318, no. 2, pp. 658-666, 2006.

[12] B. de Malafosse and V. Rakočević, "Applications of measure of noncompactness in operators on the spaces $s_{\alpha}, s_{\alpha}^{0}, s_{\alpha}^{(c)}, l_{\alpha}^{p}$, Journal of Mathematical Analysis and Applications, vol. 323, no. 1, pp. 131-145, 2006.

[13] M. Mursaleen, V. Karakaya, H. Polat, and N. Simşek, "Measure of noncompactness of matrix operators on some difference sequence spaces of weighted means," Computers \& Mathematics with Applications, vol. 62, no. 2, pp. 814-820, 2011.

[14] M. Mursaleen and A. K. Noman, "Applications of Hausdorff measure of noncompactness in the spaces of generalized means," Mathematical Inequalities \& Applications, vol. 16, no. 1, pp. 207-220, 2013.

[15] A. Wilansky, Summability Through Functional Analysis, vol. 85 of North-Holland Mathematics Studies, North-Holland, Amsterdam, The Netherlands, 1984.

[16] A. M. Jarrah and E. Malkowsky, "Ordinary, absolute and strong summability and matrix transformations," Univerzitet u Nišu. Prirodno-Matematički Fakultet, no. 17, pp. 59-78, 2003.

[17] E. Malkowsky and V. Rakočević, "The measure of noncompactness of linear operators between certain sequence spaces," Acta Scientiarum Mathematicarum, vol. 64, no. 1-2, pp. 151-170, 1998.

[18] A. Peyerimhoff, "Über ein Lemma von Herrn H. C. Chow," Journal of the London Mathematical Society, vol. 32, pp. 33-36, 1957.
[19] E. Malkowsky, "Banach algebras of matrix transformations between spaces of strongly bounded and summable sequences," Advances in Dynamical Systems and Applications, vol. 6, no. 1, pp. 91-109, 2011.

[20] R. R. Akhmerov, M. I. Kamenskiĭ, A. S. Potapov, A. E. Rodkina, and B. N. Sadovskiǔ, Measures of Noncompactness and Condensing Operators, vol. 55, Birkhäuser, Basel, Switzerland, 1992.

[21] J. M. A. Toledano, T. Dominguez Benavides, and G. Lopez Acedo, Measures of Non-Compactness in Metric Fixed Point Theory, Operator Theory, Advances and Applications, vol. 99, Birkhäuser, Basel, Switzerland, 1997.

[22] J. Banaś and K. Goebl, Measures of Noncompactness in Banach Spaces, vol. 60 of Lecture Notes in Pure and Applied Mathematics, Marcel Dekker, Inc, New York, NY, USA, 1980.

[23] E. Malkowsky and V. Rakočević, "An introduction into the theory of sequence spaces and measures of noncompactness," Zbornik Radova, vol. 9, article 17, 2000, Matematički Institut SANU, Beograd, pp. 143-234, 2000. 


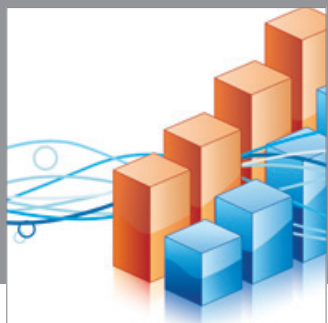

Advances in

Operations Research

mansans

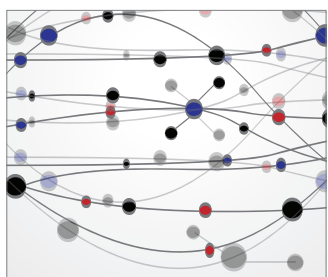

The Scientific World Journal
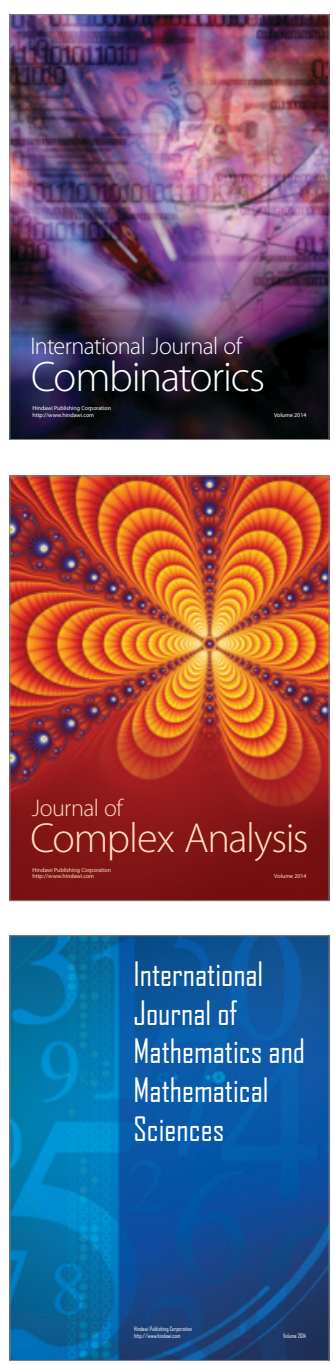
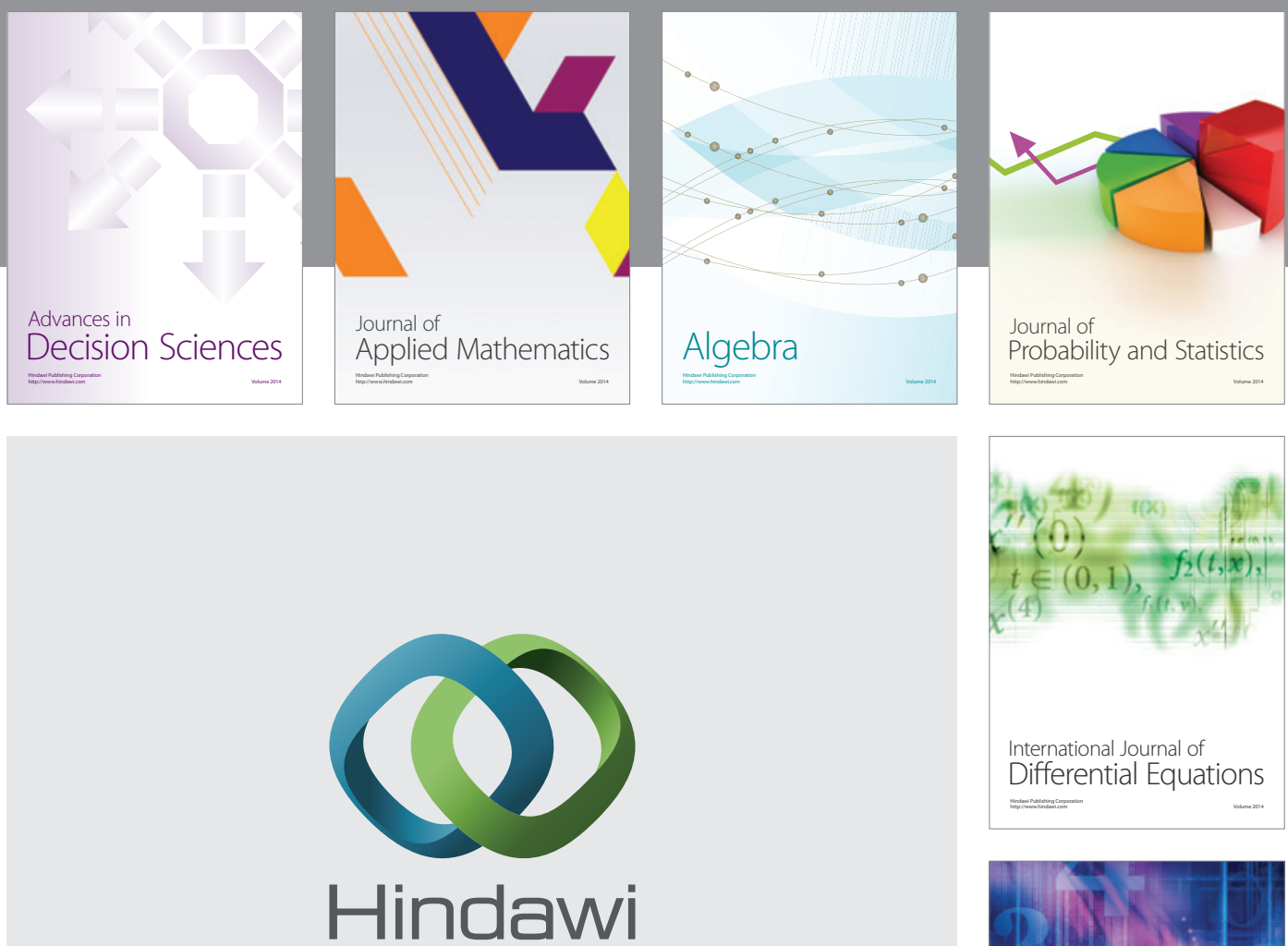

Submit your manuscripts at http://www.hindawi.com
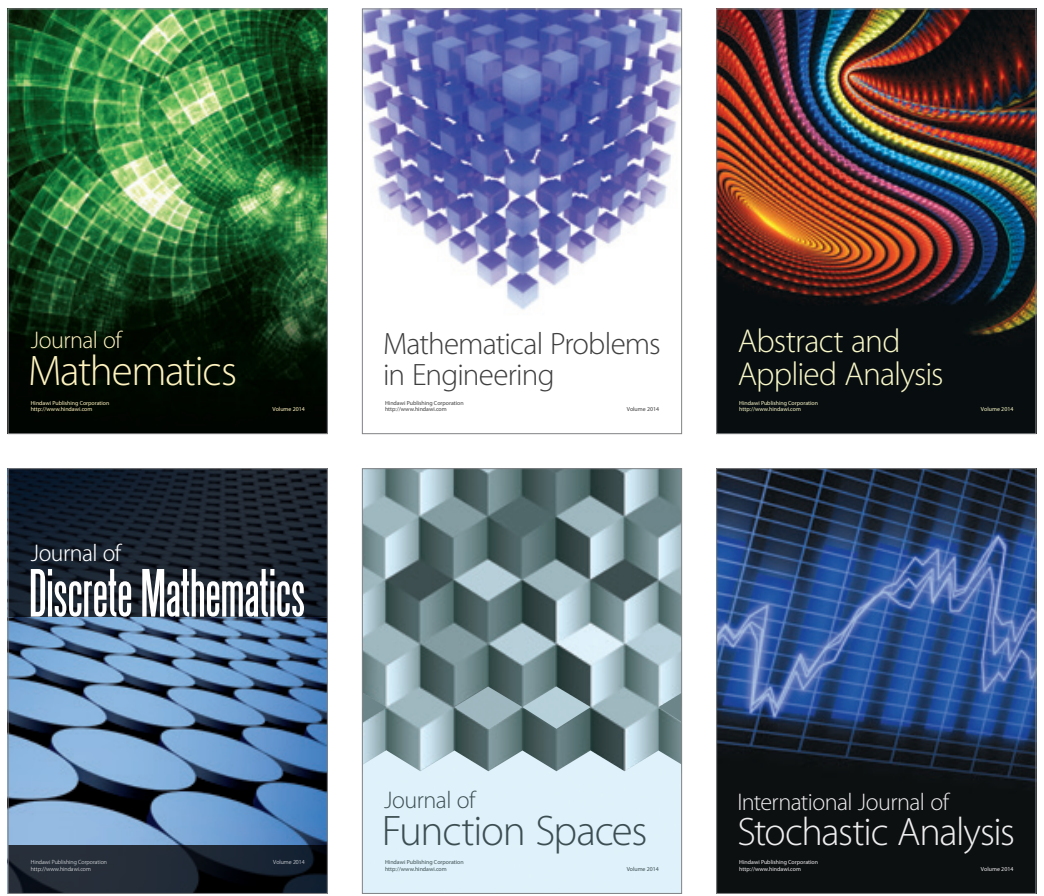

Journal of

Function Spaces

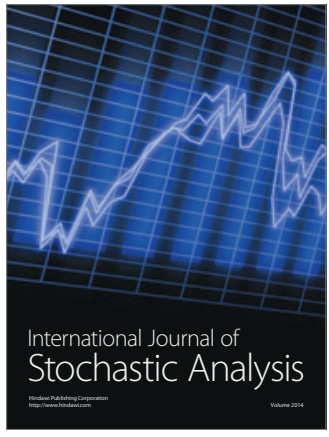

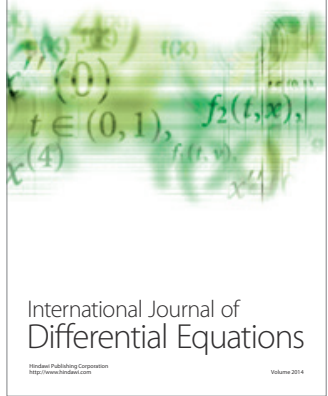
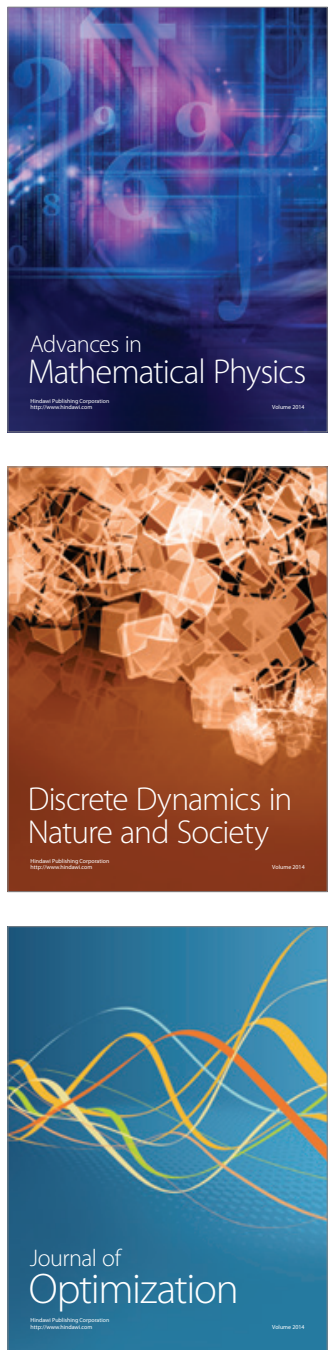